\title{
Phylogeographic Analysis of African Swine Fever Virus, Western Europe, 2018
}

\section{Mutien Garigliany, Daniel Desmecht, Marylène Tignon, Dominique Cassart, Christophe Lesenfant, Julien Paternostre, Rosario Volpe, Ann Brigitte Cay, Thierry van den Berg, Annick Linden}

Author affiliations: University of Liège, Sart Tilman, Belgium (M. Garigliany, D. Desmecht, D. Cassart, C. Lesenfant, J. Paternostre, R. Volpe, A. Linden); Sciensano Animal Health, Brussels, Belgium (M. Tignon, A.B. Cay, T. van den Berg)

DOI: https://doi.org/10.3201/eid2501.181535

In September 2018, African swine fever in wild boars was detected in Belgium. We used African swine fever-infected spleen samples to perform a phylogenetic analysis of the virus. The causative strain belongs to genotype II, and its closest relatives are viruses previously isolated in Ukraine, Belarus, Estonia, and European Russia.

A frican swine fever (ASF) is a devastating disease of domestic pigs and wild boars caused by a DNA arbovirus, African swine fever virus (ASFV), belonging to the family Asfarviridae. ASF is endemic in sub-Saharan African countries and has become more prevalent in the Caucasus region since its spread from eastern Africa to Georgia in 2007. The epizootic then spread to the surrounding countries, including the Russian Federation, and further to Belarus and Ukraine. In 2014, ASFV reached the European Union member states of Estonia, Latvia, Lithuania, and Poland; in 2016, Moldova; and, in 2017, the Czech Republic and Romania (1).

On September 13, 2018, authorities in Belgium reported that ASF had been confirmed in 2 wild boars near the village of Étalle $\left(49.6833^{\circ} \mathrm{N}, 5.6^{\circ} \mathrm{E}\right)$, in the province of Luxembourg, which is located $12 \mathrm{~km}$ from the border with France and $17 \mathrm{~km}$ from the country of Luxembourg. ASFV appears to have jumped a considerable distance from previously affected countries: $\approx 500 \mathrm{~km}$ from the border with the Czech Republic, $800 \mathrm{~km}$ from Hungary, and 1,200 km from the border with Romania. Since then, $\approx 75 \%$ of the wild boars found dead near the primordial spot have been found to be ASFV positive; a total of 96 positive results had been recorded as of October 16, 2018.

To investigate the virus, we performed initial genetic characterization using standard genotyping procedure on virus DNA directly extracted from homogenized spleen or kidney tissues of each animal. First, we obtained a segment of the $B 646 \mathrm{~L}$ gene by PCR as described by Gallardo et al. (2) and Ge et al. (3). The DNA sequence retrieved was identical in both animals (GenBank accession no. MH998358). We performed sequence alignments using ClustalW implemented in Geneious version 8.1 .8 (https:// www.geneious.com). We performed phylogenetic analysis using MEGA7 (http://www.megasoftware.net) and the Kimura 2-parameter substitution model, using the neighbor-joining method, as determined by a model selection analysis (Figure, panel A). The strain of ASFV found in Belgium clearly belonged to genotype II, which includes viruses that are circulating in both Eurasia and southern Africa.

To further define the most likely origin of this strain, we performed PCR targeting a $\approx 350$-bp fragment in the variable intergenic region between the $I 73 R$ and $I 329 L$ genes, according to Gallardo et al. (2). Again, we retrieved identical DNA sequences from both animals (GenBank accession no. MH998359). Sequence alignments revealed that the isolate ASFV/Etalle/wb/2018 from Belgium contains a 10-nt (TATATAGGAA) insertion at position 106. It is therefore a so-called intergenic region (IGR) II variant, according to the nomenclature of Gallardo et al. (2). ASFV/Etalle/wb/2018 displays 100\% identity with the sequences obtained from strains isolated in Ukraine in 2012, Belarus in 2013, Estonia in 2014, European Russia in 2015 and 2016, and China in 2018 $(3,4)$, suggesting that the strain in Belgium most likely originates from one of these countries (Figure, panel B). Conversely, this insertion is absent in strains isolated in Armenia in 2007, in Georgia in 2007, in Poland in 2015, and in Siberia, Russia, in 2017 (4). Genotyping the p72 and IGR loci is compatible with the current state of the art for ASFV molecular epidemiology but still presents intrinsic limitations. A further genome-wide genotyping approach is expected to consolidate and bring more precision to the filiation revealed here.

\section{Acknowledgments}

The authors thank Laetitia Delaval and Mathieu Franssen for technical assistance.

The field part of this study is supported by a regional grant of the Public Service of Wallonia.

\footnotetext{
About the Author

Dr. Garigliany is a professor of pathology on the Veterinary Faculty of the University of Liège, Belgium. His research activities focus on the biology of viruses and related hostpathogen interactions.
} 


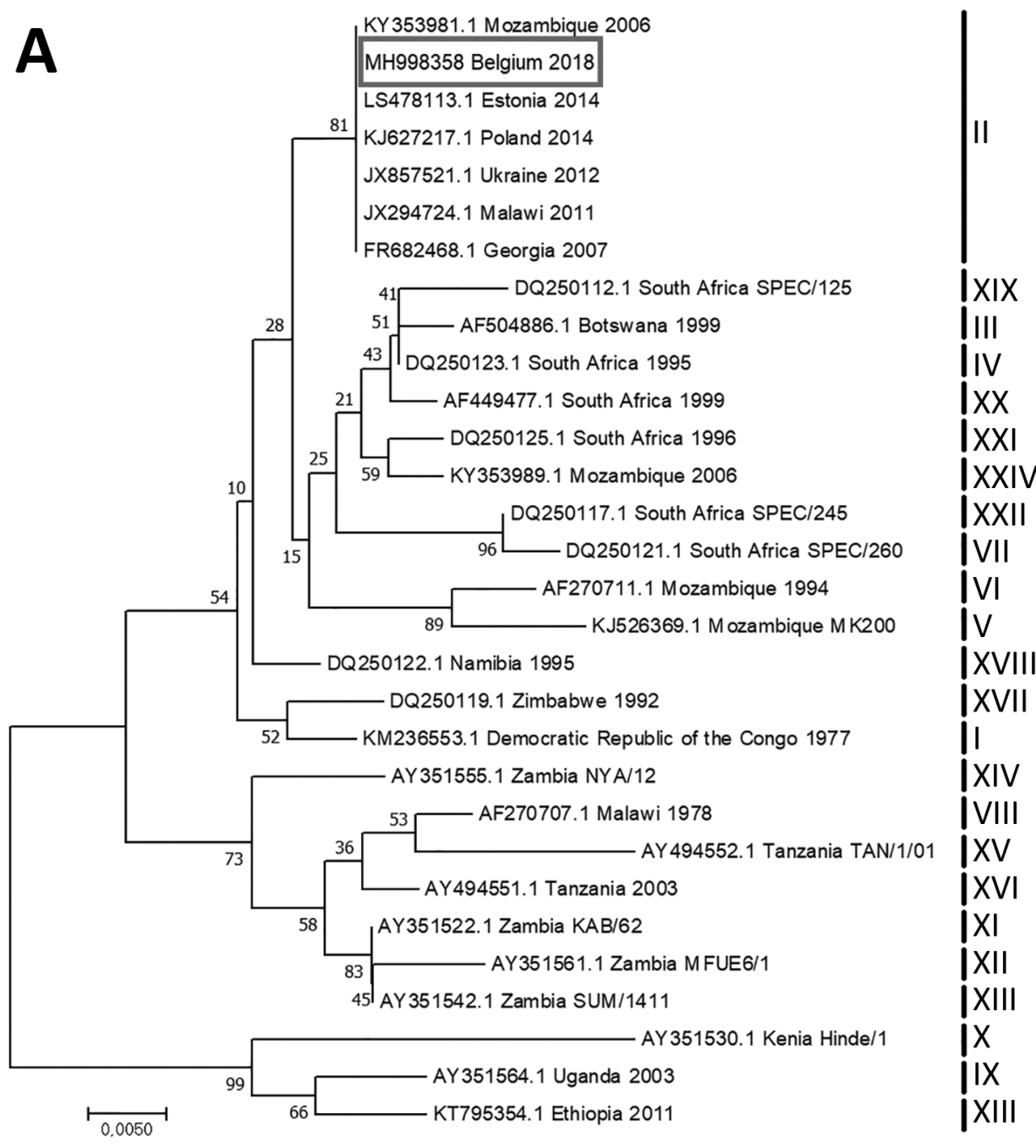

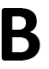

AY261364-1 Malawi 1962 KJ620043.1 Belarus 2013 KJ620037.1 Ukraine 2012 KY385893.1 Russia 2016 KY385895.1 Russia 2015 LS478113.1 Estonia 2014 MH998359 Belgium 2018 KJ620028.1 Armenia 2007 MH681419.1 Poland 2015 FR682468.1 Georgia 2007 KY982843.1 Russia 2017

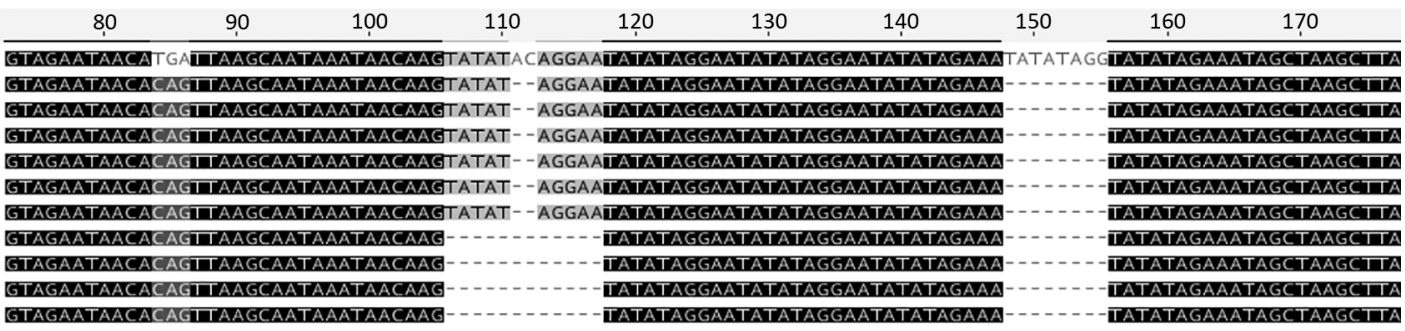

Figure. A) Evolutionary relationships of representative strains of African swine fever virus based on the neighbor-joining phylogeny of the partial p72 gene sequences. The phylogenetic analysis was performed using MEGA7 (http://www.megasoftware.net) and the Kimura 2-parameter substitution model, as determined by a model selection analysis. Bootstrap values ( $\geq 70 \%$, based on 500 replicates) for each node are given. GenBank accession numbers, country, and year of collection are indicated for each strain; for strains for which the year of collection is not known, the strain name is indicated. Corresponding genotypes are labeled IXXIV. Box indicates the African swine fever sequence from Belgium generated during this study. Scale bar indicates nucleotide substitutions per site. B) Nucleotide sequence alignment of the partial intergenic region between the $I 73 R$ and $I 329 L$ genes from representative African swine fever virus strains. GenBank accession numbers, country, and year of collection are indicated. 


\section{References}

1. Chenais E, Ståhl K, Guberti V, Depner K. Identification of wild boar-habitat epidemiologic cycle in African swine fever epizootic. Emerg Infect Dis. 2018;24:810-2. http://dx.doi.org/ 10.3201/eid2404.172127

2. Gallardo C, Fernández-Pinero J, Pelayo V, Gazaev I, Markowska-Daniel I, Pridotkas G, et al. Genetic variation among African swine fever genotype II viruses, eastern and central Europe. Emerg Infect Dis. 2014;20:1544-7. http://dx.doi.org/ 10.3201/eid2009.140554

3. Ge S, Li J, Fan X, Liu F, Li L, Wang Q, et al. Molecular characterization of African swine fever virus, China, 2018. Emerg Infect Dis. 2018;24:2131-3. http://dx.doi.org/10.3201/ eid2411.181274

4. Kolbasov D, Titov I, Tsybanov S, Gogin A, Malogolovkin A. African swine fever virus, Siberia, Russia, 2017. Emerg Infect Dis. 2018;24:796-8. http://dx.doi.org/10.3201/eid2404.171238

Address for correspondence: Daniel Desmecht, University of Liège, Pathology, Faculty of Veterinary Medicine, Sart Tilman B43, Liège 4000, Belgium; email: daniel.desmecht@ulg.ac.be

\section{Inaccurate Multilocus Sequence Typing of Acinetobacter baumannii}

\section{Santiago Castillo-Ramírez, Lucía Graña-Miraglia}

Author affiliation: Programa de Genómica Evolutiva, Centro de Ciencias Génomicas, Universidad Nacional Autónoma de México, Cuernavaca, Mexico

DOI: https://doi.org/10.3201/eid2412.180374

Multilocus sequence typing has been useful for genotyping pathogens in surveillance and epidemiologic studies. However, it cannot reflect the true relationships of isolates for species with very dynamic genomes. Using a robust genome phylogeny, we demonstrated the limitations of this method for typing Acinetobacter baumannii.

A $\mathrm{n}$ adequate genotyping system is of paramount importance for infectious disease epidemiology. Two decades ago, the multilocus sequence typing (MLST) scheme was proposed as a genotyping method (1), and today, because of its reproducibility and portability, MLST schemes are available for many human pathogens (2). MLST has been instrumental in increasing understanding of the epidemiology and population structure of many bacteria.
Acinetobacter baumannii, a major source of nosocomial infections, is no exception, and 2 MLST schemes (Oxford and Pasteur) have been established for this species $(3,4)$. Each scheme uses just 7 loci and, therefore, only samples a small fraction of the chromosome, which could be a serious issue for genotyping species with highly variable genomes. Some studies have shown that $A$. baumannii has both high gene content variation (5) and substantial levels of recombination (6).

We revisited one of the most comprehensive genome datasets of A. baumannii (5) to construct a robust phylogeny to show that sequence type (ST) assignation in both MLST schemes does not reflect true relationships among isolates of this species. This dataset of $>80$ genomes covers 36 different STs according to the Oxford scheme (STox) and 19 different STs according to the Pasteur scheme (STp) (Appendix Table 1, https://wwwnc.cdc.gov/EID/article/25/1/18-0374-App1. pdf). We constructed a concatenated alignment of 574 orthologous genes and conducted statistical model selection as in a previous study (5) and, on that alignment, constructed a maximum-likelihood phylogeny by PhyML (7).

The 2 schemes showed different levels of resolution. Although in many instances a single STp had just 1 equivalent STox, 2 STs exist in the Pasteur scheme that encompass many Oxford STs (Appendix Figure, red branches). For instance, under the Pasteur scheme, STp2 represents $>15$ STs in the Oxford scheme and STp1 encompasses 5 STox. Thus, the Pasteur scheme seems to have considerably less resolution than the Oxford scheme to distinguish isolates. The Pasteur scheme's lack of resolution was not insignificant, however. STp2 comprises 43 isolates (approximately half of our dataset) showing considerable levels of genetic variation according to our phylogeny, but according to this MLST scheme, they constitute just 1 genotype.

Many of the STs in either scheme formed coherent (monophyletic) groups in our phylogeny. However, we recorded some clear exceptions in which isolates from some STs did not form monophyletic groups, that is, isolates with the same ST did not cluster. The most striking case is STox208 (orange tips in the phylogeny), where there are 2 well-defined groups with several isolates each and an extra isolate not close to either of those well-defined groups. We also noted that the 2 STox 455 isolates did not cluster and are located far apart on the tree (green tips). Additionally, 1 of the STox369 isolates did not fall within the ST369 group (blue tips). These 3 examples show that the Oxford MLST does not accurately reflect the relationships among the isolates. Also notable is that, although for the Oxford scheme 36 STs are represented in this dataset, only 16 of them have $\geq 2$ isolates and therefore only in these STs could we detect problems with the clustering within any given ST. Thus, 3 of these 16 STs did not cluster the isolates properly inasmuch as these STs were polyphyletic. In summary, for the Oxford scheme we demonstrated that some STs form polyphyletic groups 